\title{
Id 2 gene is a transcriptional target of the protein complex mutant p53/E2F1
}

\author{
Giulia Fontemaggi, ${ }^{1-3}$ Stefania Dell'Orso, ${ }^{1,2}$ Paola Muti, ${ }^{4}$ Giovanni Blandino ${ }^{1,2, *}$ and Sabrina Strano ${ }^{5}$
}

\begin{abstract}
'Translational Oncogenomics Unit; ${ }^{2}$ Rome Oncogenomic Center (ROC); and ${ }^{4}$ Scientific Direction; Regina Elena Cancer Institute; Rome, Italy; ${ }^{3}$ General Pathology Section; Department of Clinical and Experimental Medicine; Perugia University; Perugia, Italy; ${ }^{5}$ Molecular Chemoprevention Group; Scientific Direction; Regina Elena Cancer Institute; Rome, Italy
\end{abstract}

It has been established that developmental regulators play a direct role in driving aspiring cancer cells towards a malignant phenotype. Inhibitors of DNA-binding/ differentiation family of proteins are dominant negative regulators of basic helix-loop-helix (bHLH) transcription factors. They play a key role during development, preventing cell differentiation while inducing cell proliferation. They are poorly expressed in adult life but can be reactivated in tumorigenesis leading to alteration in cell cycle progression, senescence and apoptosis. ${ }^{1,2}$

We have recently shown that mutant $\mathrm{p} 53$ proteins specifically induce Id 4 expression in breast cancer cells. ${ }^{3}$ Mutant $\mathrm{p} 53$ proteins, usually expressed at markedly elevated levels in cancer cells, are associated with drug resistance and greater tumorigenicity. ${ }^{4-7}$ We have shown that Id 4 protein expression is enriched in breast cancer (BC) tissues showing $\mathrm{p} 53$ overexpression (predictive of the presence of mutant $\mathrm{p} 53) .^{3}$ The activation of Id 4 promoter is driven by the transcriptional competent protein complex mutant p53/E2F1/p300 and leads to an increase of the angiogenic potential of mutant p53-carrying tumor cells. ${ }^{3}$

We explored whether additional Id family members, besides Id4, were regulated by mutant p53 and we found that Id2 expression was increased following p53R175H induction in H1299 lung adenocarcinoma inducible cells. The increase was more pronounced in the presence of a DNA damaging agent, like cisplatin (Fig. 1A). Id 2 induction was also observed after infection with a retroviral vector expressing mutant p53R175H (Fig. 1B). To evaluate whether endogenous mutant p53 controls Id 2 expression, we depleted $\mathrm{p} 53$ expression in $\mathrm{SKBr} 3$ breast cancer cell line, carrying p53R175H. As shown in Figure 1C Id2 expression is reduced by $35 \%$ after mutant p53 depletion in proliferating cells. We then analyzed Id 2 promoter occupancy by Chromatin Immunoprecipitation (ChIP), amplifying a region upstream to Id 2 transcription start site (TSS), which contained binding sites for E2F (E2FF and CDE elements), NF-Y (CCAAT), NFкB and Sp1 (outlined in Fig. 1D). We found that mutant p53, p65 (NFKB), E2F1 and p300 are recruited to this promoter region (Fig. 1E) in proliferating SKBr3 cells. Given that we have previously demonstrated that mutant $\mathrm{p} 53$ is recruited to $\mathrm{Id} 4$ promoter regions by DNA-binding transcription factors, 3,8 such as E2F1 and NEKB, we analyzed by ReChIP experiments whether these last mediate mutant $\mathrm{p} 53$ recruitment to Id 2 promoter too. As shown in Figure $1 \mathrm{~F}$ mutant p53 is strongly associated to E2F1 and p300 on Id2 promoter.

We then analyzed Id 2 mRNA levels in mutant p53-carrying cell lines after sub-lethal doses of DNA damage and we observed a strong induction in all the analyzed cell lines (Fig. 1G). This induction is mutant p53-dependent, as p53 depletion in SW480 cells (carrying p53R273H/ P309S) reduces the induction of Id2 transcript (Fig. 1H). ChIP analyses in SW480 untreated or after DNA damage showed that mutant p53 is bound with similar efficiency to Id 2 promoter in both conditions, while the binding of p 65 and, more markedly, that of E2F1 is increased after DNA damage. ReChIP assay showed that mutant $\mathrm{p} 53$ is associated with p 65 in untreated cells, while during DNA damage mutant p53 is strongly and specifically associated only with E2F1.
ReChIP data obtained in SKBr3 and SW480 cells suggest that mutant p53 cooperates with E2F1 in the control of Id 2 promoter. A previous report by Yan et al. showed that Id 2 is repressed by mutant p53 in proliferating SW480 cells. ${ }^{9}$ Here we show that under DNA damaging condition the mutp53/E2F1 complex positively controls Id2 expression in SW480 cells. These apparently contradictory findings might reflect the assembly of selective protein complexes, containing mutant $\mathrm{p} 53$, DNA-binding transcription factors and co-activators, such as p300 or PCAF, or co-repressors, such as HDACs, whose output ranges from activation to repression.

\section{Acknowledgements}

This work was supported by the European Community (EC) FP6 "Active p53" consortia. This publication reflects the authors' views and not necessarily those of the EC. The EC is not liable for any use that may be made of the information contained herein. The support given by AIRC-ROC to the oncogenomic platform, AIRC to G.B., S.S., Lega Italiana Tumori to S.S., Fondazione Veronesi, Ministero della Sanità and Alleanza contro il cancro is greatly appreciated.

\section{References}

1. Perk J, et al. Nat Rev Cancer 2005; 5:603-14

2. Lasorella A, et al. Oncogene 2001; 20:8326-33.

3. Fontemaggi G, et al. Nat Struct Mol Biol 2009; 16:1086-93.

4. Oren M, et al. Cold Spring Harb Perspect Biol 2010; 2:1107.

5. Brosh R, et al. Nat Rev Cancer 2009; 9:701-13.

6. Bossi G, et al. Cell Cycle 2008; 7:1870-9.

7. Di Agostino S, et al. Cell Cycle 2008; 7:3440-7.

8. Di Agostino S, et al. Cancer Cell 2006; 10:191-202.

9. Yan W, et al. Cancer Res 2008; 68:6789-96. 


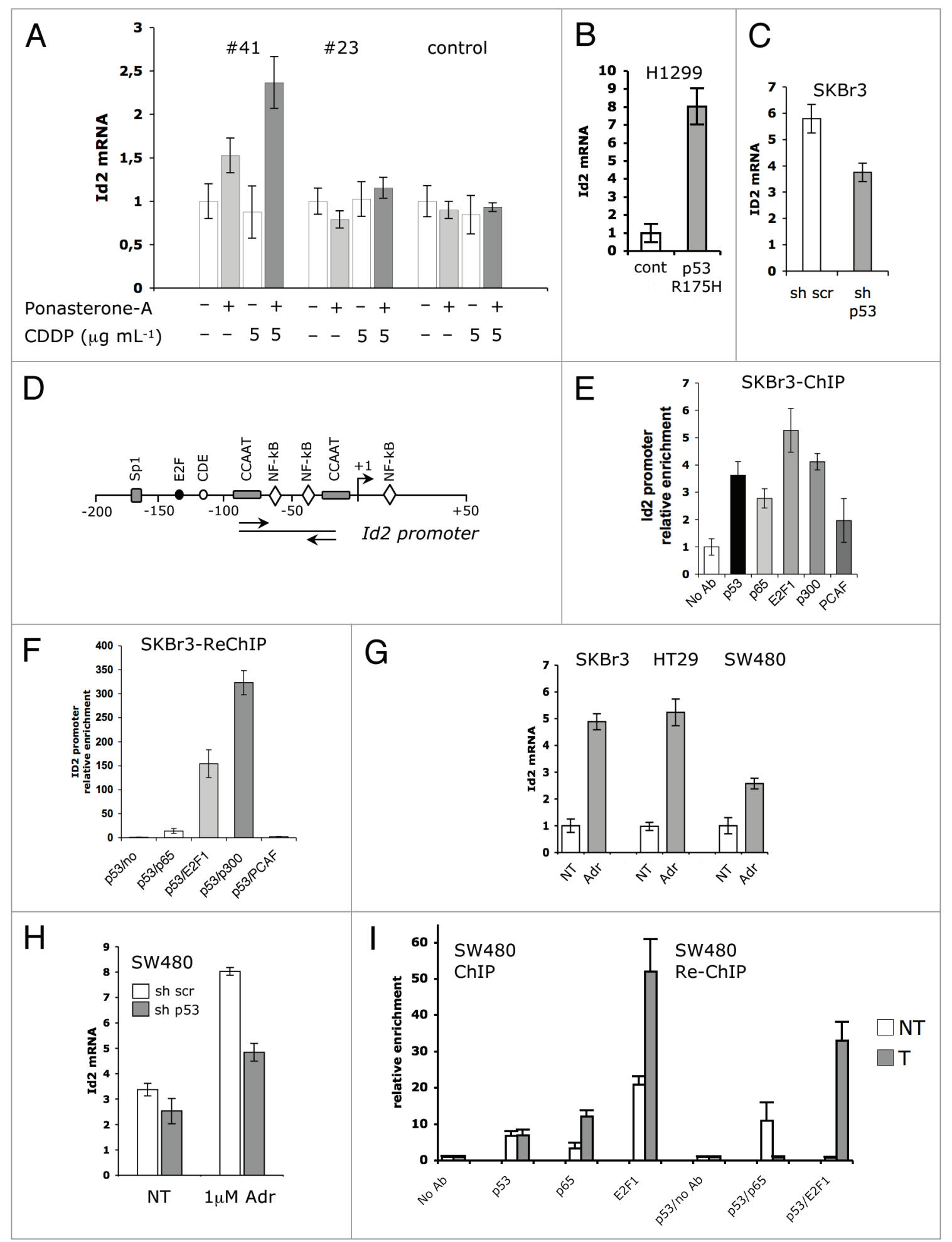

Figure 1. For figure legend, see page 2466. 
Figure 1 (See previous page). Id 2 is transcriptionally induced by mutant $p 53$. (A) Quantitative RT-PCR (qRT-PCR) analysis of Id $2 \mathrm{mRNA}$ expression on clone 41 (expressing inducible p53R175H), clone 23 (expressing inducible wild-type p53) and control clone (carrying the empty vector) in presence or absence of Ponasterone $A$ and/or cisplatin. The following primers for Id 2 transcript detection were used: forward, 5'-GAC CAC CCT CAA CAC GGA TA; reverse, 5'-CAC ACA GTG CTT TGC TGT CA. Relative Id 2 mRNA levels were calculated by normalization for the amount of GAPDH transcript present in the RNA preparations. (B and C) qRT-PCR analysis of Id 2 mRNA expression in (B) $\mathrm{H} 1299$ cells infected with a retroviral vector expressing p53R175H, (C) $\mathrm{SKBr} 3$ cells depleted of mutant $\mathrm{p} 53$ expression. (D) Schematic representation of Id 2 promoter. (E) Cross-linked chromatin derived from proliferating $\mathrm{SKBr} 3$ cells was subjected to ChIP using the indicated antibodies. The occupancy of Id2 promoter was analyzed by qPCR using the following primers: forward 5'-GAA CGC GGA AGA ACC AAG; reverse 5'-TTC CCT TCG TCC CCA TTG. (F) Four additional aliquots of chromatin immunoprecipitated with antip53 antibody were eluted and re-immunoprecipitated with No Ab (as negative control) or with antibodies directed against p65, E2F1, p300 and PCAF. (G) qRT-PCR analysis of Id 2 expression in SKBr3, HT29 and SW480 cells following treatment with adriamycin (1 $\mu$ M) for 36 hours. (H) qRT-PCR analysis of Id 2 mRNA expression in SW480 cells depleted of mutant p53 expression following treatment with adriamycin (1 $\mu \mathrm{M})$ for 36 hours. (I) Cross-linked chromatin derived from SW480 cells treated (T) or not (NT) with adriamycin (1 $\mu \mathrm{M})$ for 36 hours was subjected to ChIP using the indicated antibodies. Three additional aliquots of chromatin immunoprecipitated with anti-p53 antibody were eluted and re-immunoprecipitated with No Ab (as negative control) or with antibodies directed against $\mathrm{p} 65$ and E2F1.

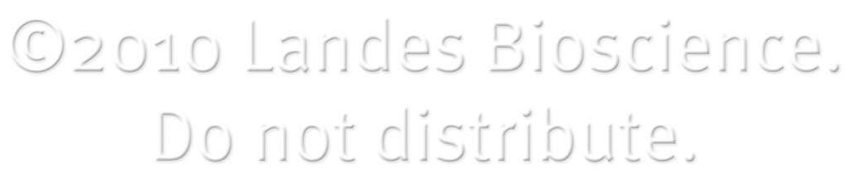

